\title{
Metabolic disassembler for understanding and predicting the biosynthetic units of natural products
}

\author{
Kohei Amano', Tsubasa Matsumoto ${ }^{1}$, Kenichi Tanaka², Kimito Funatsu ${ }^{2}$ and Masaaki Kotera2 ${ }^{2 *}$
}

\begin{abstract}
Background: Natural products are the source of various functional materials such as medicines, and understanding their biosynthetic pathways can provide information that is helpful for their effective production through the synthetic biology approach. A number of studies have aimed to predict biosynthetic pathways from their chemical structures in a retrosynthesis manner; however, sometimes the calculation finishes without reaching the starting material from the target molecule. In order to address this problem, the method to find suitable starting materials is required.

Results: In this study, we developed a predictive workflow named the Metabolic Disassembler that automatically disassembles the target molecule structure into relevant biosynthetic units (BUs), which are the substructures that correspond to the starting materials in the biosynthesis pathway. This workflow uses a biosynthetic unit library $(\mathrm{BUL})$, which contains starting materials, key intermediates, and their derivatives. We obtained the starting materials from the KEGG PATHWAY database, and 765 BUs were registered in the BUL. We then examined the proposed workflow to optimize the combination of the BUs. To evaluate the performance of the proposed Metabolic Disassembler workflow, we used 943 molecules that are included in the secondary metabolism maps of KEGG PATHWAY. About $95.8 \%$ of them (903 molecules) were correctly disassembled by our proposed workflow. For comparison, we also implemented a genetic algorithm-based workflow, and found that the accuracy was only about $52.0 \%$. In addition, for $90.7 \%$ of molecules, our workflow finished the calculation within one minute.

Conclusions: The Metabolic Disassembler enabled the effective disassembly of natural products in terms of both correctness and computational time. It also outputs automatically highlighted color-coded substructures corresponding to the BUs to help users understand the calculation results. The users do not have to specify starting molecules in advance, and can input any target molecule, even if it is not in databases. Our workflow will be very useful for understanding and predicting the biosynthesis of natural products.
\end{abstract}

Keywords: Natural products, Biosynthetic pathway, Starting material

\section{Background}

Plants biosynthesize various natural products that protect them from being eaten by herbivores. Herbivore insects can detoxify these phytotoxic molecules and can biosynthesize various molecules including pheromones. Fungi are also a source of valuable natural products such as antibiotics. Secondary metabolites are species-specific natural products that are not directly involved in the

\footnotetext{
*Correspondence: maskot@chemsys.tu-tokyo.ac.jp

${ }^{2}$ Department of Chemical System Engineering, School of Engineering, The

University of Tokyo, 7-3-1 Hongo, Bunkyo-ku, Tokyo 113-8656, Japan

Full list of author information is available at the end of the article
}

species survival. Secondary metabolites and their derivatives are used widely in medicines, cosmetics, and agriculture. For example, penicillin, which was discovered by Fleming in 1928 [1], was widely used as an antibiotic against infectious diseases, and avermectin, which was discovered by Omura in 1979 [2], is widely used as an anti-parasite drug for pets.

Identifying the biosynthetic route of natural products is not only of pharmacological interest, but is also required by engineers and biologists who use synthetic biology techniques [3-8]. Synthetic biologists aim to synthesize desired molecules enzymatically by introducing foreign

(c) The Author(s). 2019 Open Access This article is distributed under the terms of the Creative Commons Attribution 4.0 International License (http://creativecommons.org/licenses/by/4.0/), which permits unrestricted use, distribution, and 
genes into an organism such as Saccharomyces cerevisiae. The biosynthesis need not necessarily mimic the actual biosynthetic pathway of the target molecule, but knowing the actual metabolic pathway can be very useful when constructing the pathway in a model organism.

However, although the chemical structures of a large number of metabolites are known, the metabolic pathways that lead to their synthesis are not yet known. Moreover, the recent development of high-throughput sequencers and metabolome analysis technology has revealed a large number of natural products for which the biosynthetic metabolic pathways are unknown. Currently, the identification of relevant enzymes in a putative pathway is carried out by trial and error based on knowledge and experience; therefore, an information science-based approach is highly desirable.

A large variety of natural products are produced from a limited variety of starting materials [9]. In this paper, we define a biosynthetic unit (BU) as a chemical substructure that is part of the starting material. Natural products can be disassembled into the same BUs or into several different BUs to help elucidate their biosynthetic pathways.

In organic synthesis, retrosynthetic analysis is used to design the synthetic route of a target molecule. The retrosynthetic analysis method uses the target molecule and constructs the route backward from the target molecule to the starting material. A similar approach can be applied to metabolic pathway prediction; however, the number of iterations required to generate all virtual reactions can easily explode, making it difficult to find an optimal solution in a finite time. In a previous study, machine learning was applied to decide whether a pair of metabolite molecules could form an enzymatic reaction, thereby showing the potential to predict the metabolic pathway [10]. Another previous study [11] is based on similarity search to find potential precursors, i.e., the compounds that are possibly

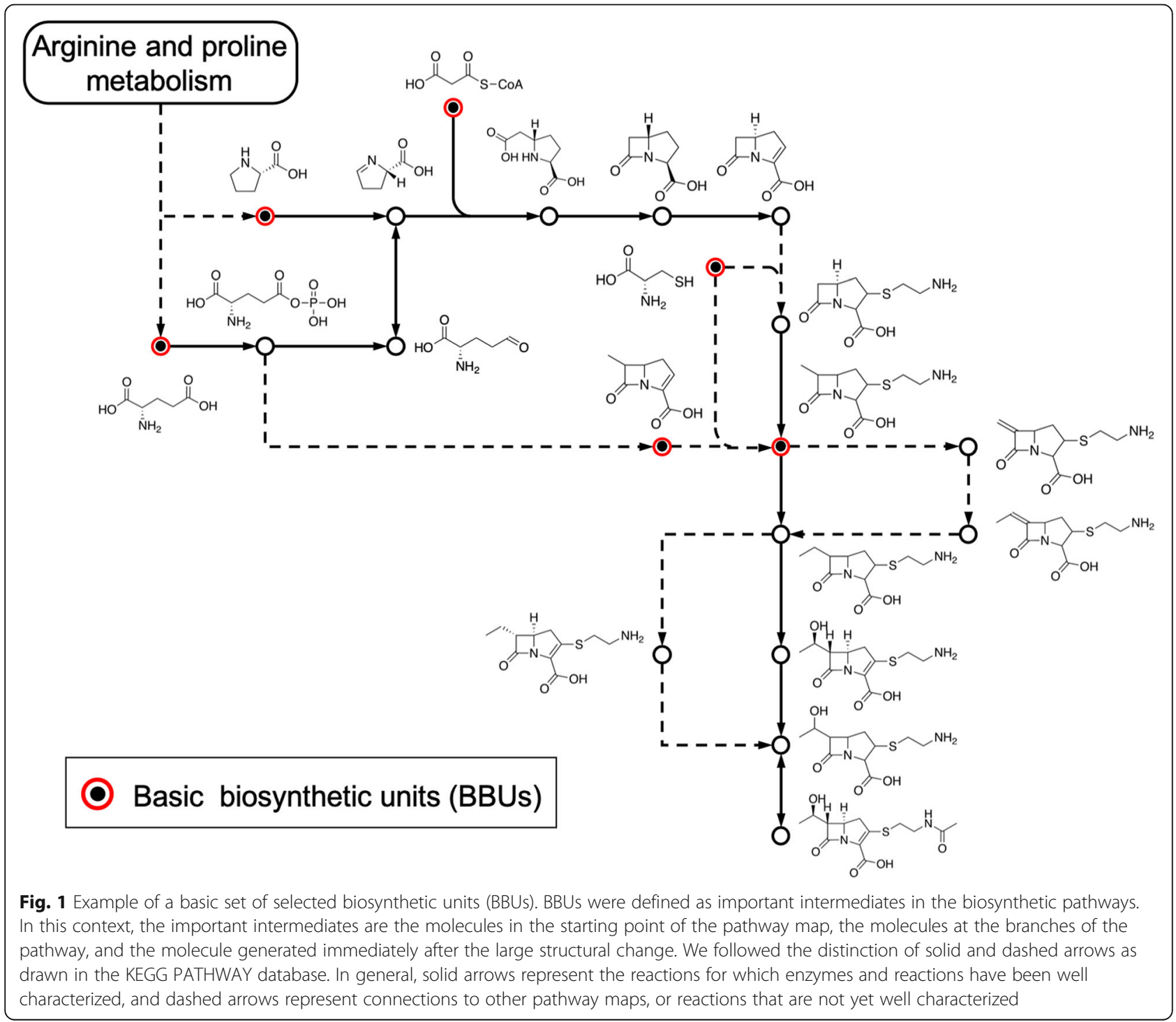


reachable in artificially designed pathway in one step. However, these approaches only deal with one-step reactions in the metabolic pathways, and therefore do not consider the putative intermediates between the target molecule and the starting material. In other studies, retrosynthetic approach often failed to reach the starting material $[3-8,12]$. To use the retrosynthetic approach for natural product biosynthesis, it is important to develop a workflow that can identify the appropriate starting materials.

In this study, we developed a workflow named the Metabolic Disassembler to automatically identify the BUs that corresponded to the starting materials of a given natural product. One of the important characteristics is that our method "disassembles" a given molecule into substructures that imply the starting materials. The aim was to provide support for identifying biosynthesis pathways. We evaluated the performance of the Metabolic Disassembler using datasets of secondary metabolites acquired from the Kyoto Encyclopedia of Genes and Genomes (KEGG) database [13], and showed the effectiveness of the workflow in terms of both accuracy and computational time. The users of the Metabolic Disassembler do not have to specify starting molecules in advance, and can input any target molecule, even if it is not in databases.

\section{Methods}

\section{Chemical structures of natural products}

The goal of the Metabolic Disassembler is not to construct artificial pathways, but is to know the actual metabolic pathways of natural products. In order to achieve our goal, compounds and reactions in primary (central) metabolism are not needed. Rather, they are a hindrance to the calculation. Also, if a secondary metabolite is not associated with known biosynthetic pathway, no one would estimate if the prediction is true. Therefore, we need to collect secondary metabolites for which biosynthetic pathways are already known. We surveyed databases including MetaCyc [14] and Rhea [15], and concluded that KEGG [13] (as of November 2018) is the one that stores necessary and sufficient amount of information.

In KEGG, metabolic compounds are given identifiers that contain the letter " $C$ " and a five-digit number (e.g., "C00078" for L-tryptophan). We also used KEGG PATHWAY secondary metabolism maps to evaluate the accuracy of disassembly. In KEGG PATHWAY, secondary metabolism is classified as 1.9 "Metabolism of terpenoids and polyketides" (hereafter referred to as the 1.9 class) and 1.10 "Biosynthesis of other secondary metabolites" (hereafter referred to as the 1.10 class). The 1.9 class contains a group of molecules that are biosynthesized through mevalonate or non-mevalonate pathways, and BUs in these pathways are mostly limited to $C_{2}$ and $C_{5}$ units. This means that it is not difficult to identify these BUs because they are already quite obvious, and it is more important to analyze how the BUs form complex rings. Therefore, in this study, we focused on the 1.10 class, which contains 1111 molecules in 28 metabolic pathway maps. Molecules

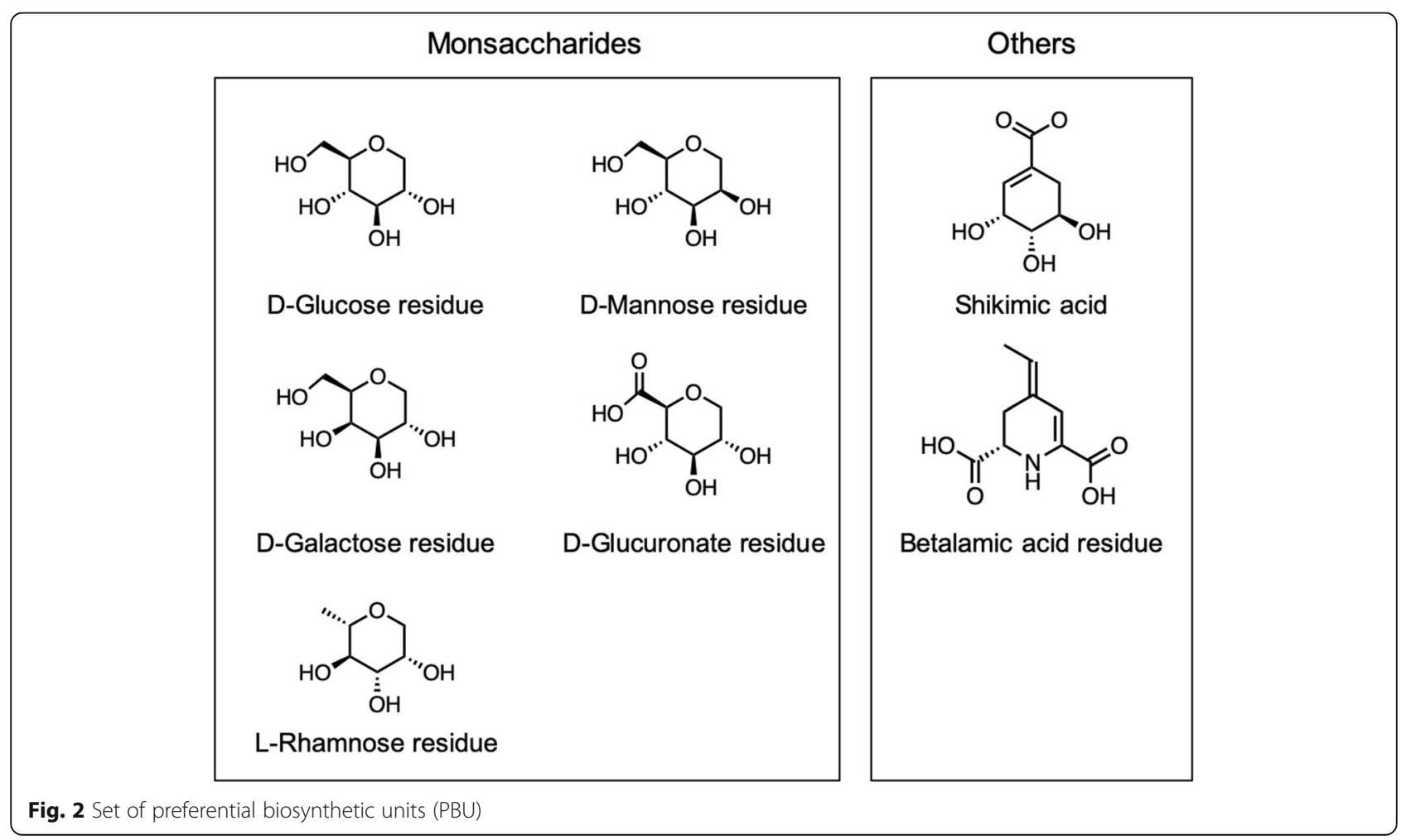


that had an " $R$ " group, indicating they contained undefined structures, caused errors when using RDKit [16], and therefore were not used. The remaining 943 molecules were used in this study. Note that we used KEGG just because it covers all known pathways, and used KEGG PATHWAY just to test the performance. We designed the Metabolic Disassembler so that the users can input any molecule even if it is not found in any database. Note again that we did not focus on the primary (central) metabolites, because all secondary metabolites originate from the starting compounds in the secondary pathway maps. Therefore, in order to find the starting materials of unknown secondary metabolites, it is more practical to use them, rather than the primary (central) metabolites.

\section{Chemical structure manipulation and computing environment}

We implemented our application software using Python programming language (version 3.6.6) on Anaconda3 (version 5.3.0) [17], NetworkX (version 2.2) [18], RDKit (version 2018.09.1.0) [16], Pycairo (version
1.18.0) [19], and CairoSVG (version 2.2.1) [20]. NetworkX is a graph calculation library that we used to produce the chemical structure graphs. RDKit is used widely in chemoinformatics, and we used it when retrieving chemical structures and conducting maximum common substructure searches. Pycairo and CairoSVG were used to render images of the molecules. The chemical structure of a molecule was represented as a chemical graph with atoms as nodes and chemical bonds as edges. Chemical structures described in MDL Molfile V2000 [21] were retrieved using RDKit and converted to chemical graphs using NetworkX. All calculations were conducted on a computer with an Intel Core i7-9700K $3.6 \mathrm{GHz} \mathrm{CPU}$ and $16 \mathrm{~GB}$ RAM in Windows 10 OS.

\section{Generating a biosynthetic unit library (BUL)}

Some minimal units for the biosynthesis of natural products are known, including the acetate- $\mathrm{C}_{2}$ unit in the malonic acid pathway and the $\mathrm{C}_{5}$ unit in the isoprenoid pathway. The goal of this study was to

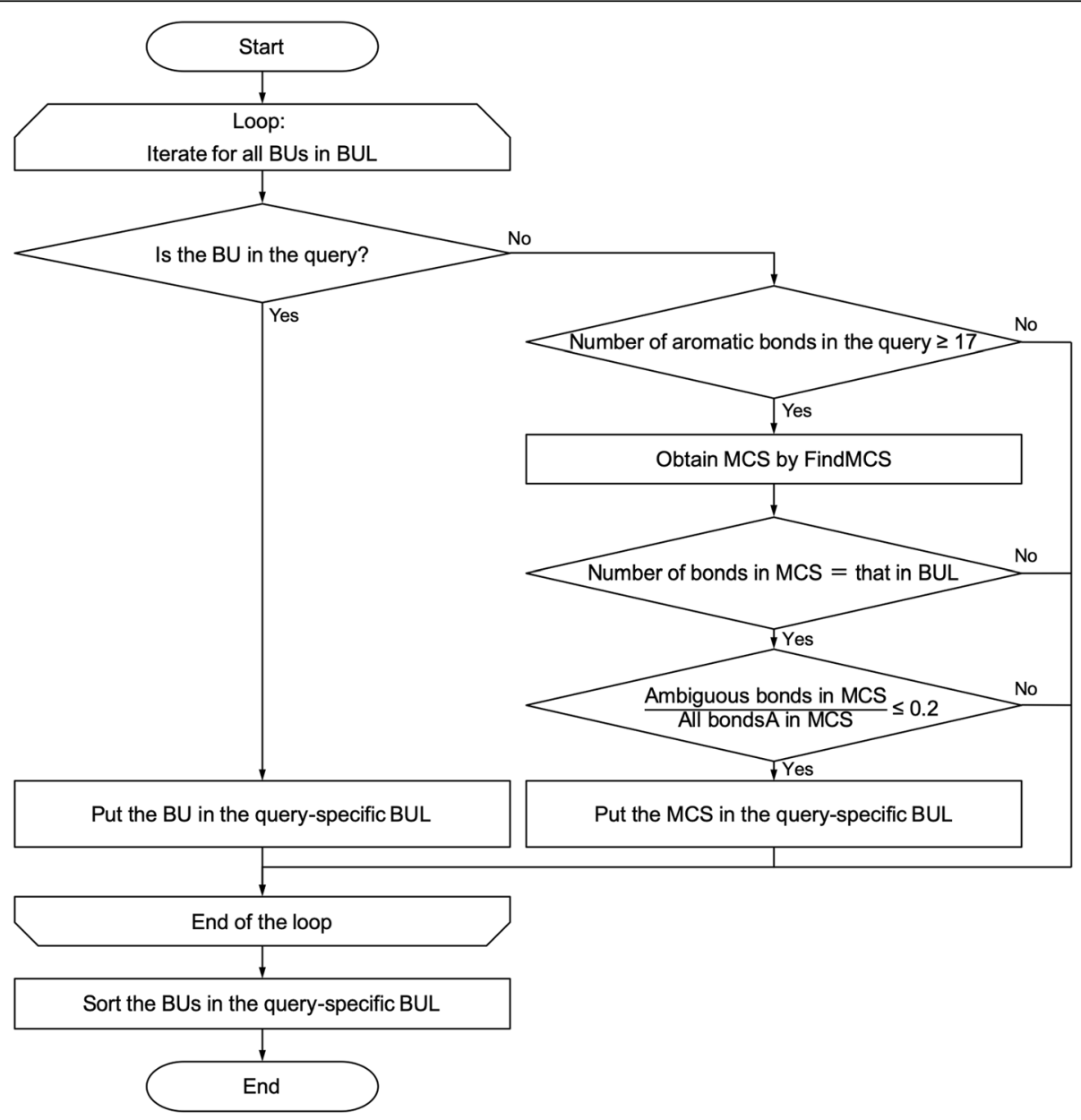

Fig. 3 The flowchart for generating a query-specific biosynthetic unit library (BUL) 
(a)

\section{Target}

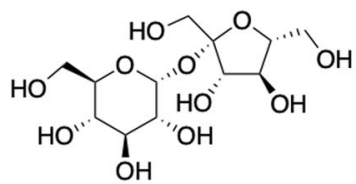

Sucrose<smiles>OC[C@H]1O[C@H](O)[C@@H](O)[C@H](O)[C@H]1O</smiles><smiles>OC[C@H]1O[C@H](O)[C@@H](O)[C@H](O)[C@H]1O</smiles><smiles>OC[C@H]1O[C@H](O)C(O)[C@@H](O)[C@H]1O</smiles>

D-Glucose

True<smiles>OC[C@H]1OC(O)[C@H](O)[C@@H](O)[C@H]1O</smiles>

Queries HO" $\mathrm{OH}$

(b)<smiles>N/C(=C/c1c[nH]c2ccccc12)C(=O)O</smiles>

1<smiles>O=C(O)c1[nH]c(C(=O)O)c(-c2c[nH]c3ccccc23)c1-c1c[nH]c2ccccc12</smiles>

2

Fig. 4 Distinguishing stereoisomers and double and aromatic bonds. (a) Example of distinguishing stereoisomers by RDKit.D-glucose with unclear stereoisomerism at 1-OH and a-D-glucose were regarded as being included in the target structure, whereas $\beta$-D-Glucose and a-D-Mannose were not. (b) Example of distinguishing double and aromatic bonds. Structure 1 was not regarded as being included in Structure 2 when using the HasSubstructMatch method in RDKit, because it distinguishes double and aromatic bonds (in red)

identify the starting materials and to provide information that can be used to predict the biosynthesis pathway; therefore, it is more useful practically to deal with substructures that are larger than the minimal units, which correspond to the metabolites located near the boundary area of primary and secondary metabolism. We defined three types of biosynthetic units (BUs), basic BUs (BBUs), derivative BUs (DBUs), and preferential BUs (PBUs), and stored them in a biosynthetic unit library (BUL).

The BBUs were defined as important intermediates in biosynthetic pathways and included the molecules

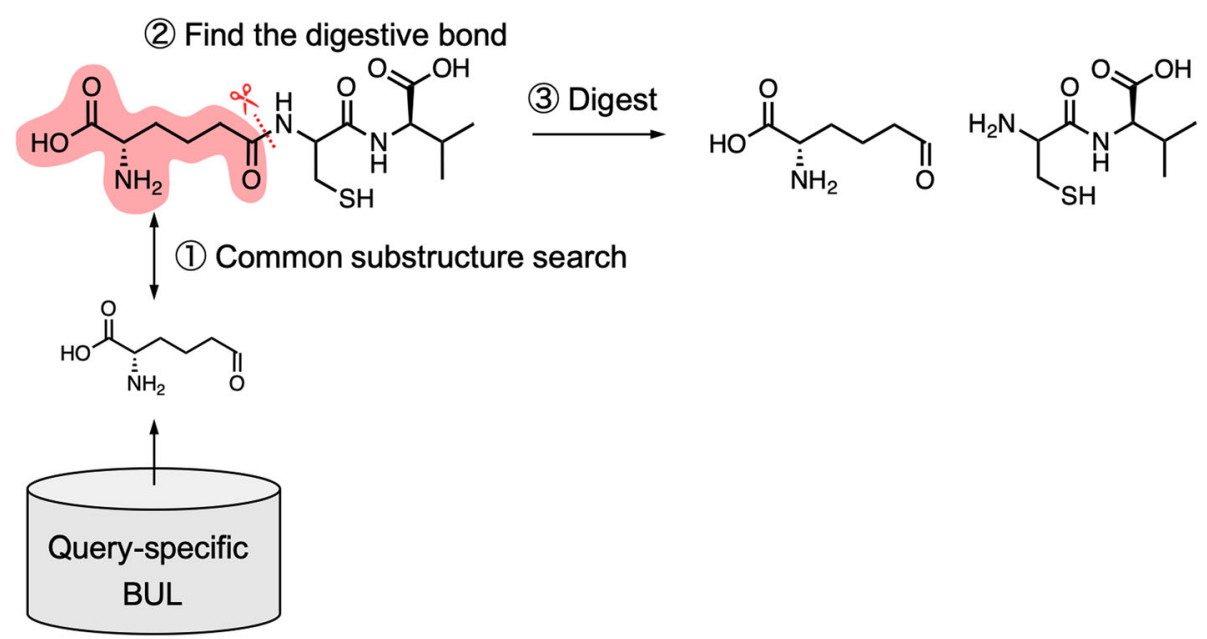

Fig. 5 Example of the fragmentation process. One of the BUs is used in a substructure search against the target molecule to identify the bond to digest. Splitting the identified bond yields fragmentation 
in the starting point of a pathway map, the molecules at the branches of a pathway, and the molecule generated immediately after a large structural change. Examples of BBUs are shown in Fig. 1. The DBUs were identified as derivatives of BBUs by applying 14 chemical transformation rules (i.e., dehydroxylation, decarboxylation, deamination, decarbonylation, oxidative deamination, amino transfer, dentrolation, dephoshorylation, dihidrogenation/de-dihydrogenation, de-CoA, denucleotidylation, ring opening, ring closure, and hydrolysis). We obtained a set of 765 BUs from 257 BBUs and 542 DBUs. The final number was

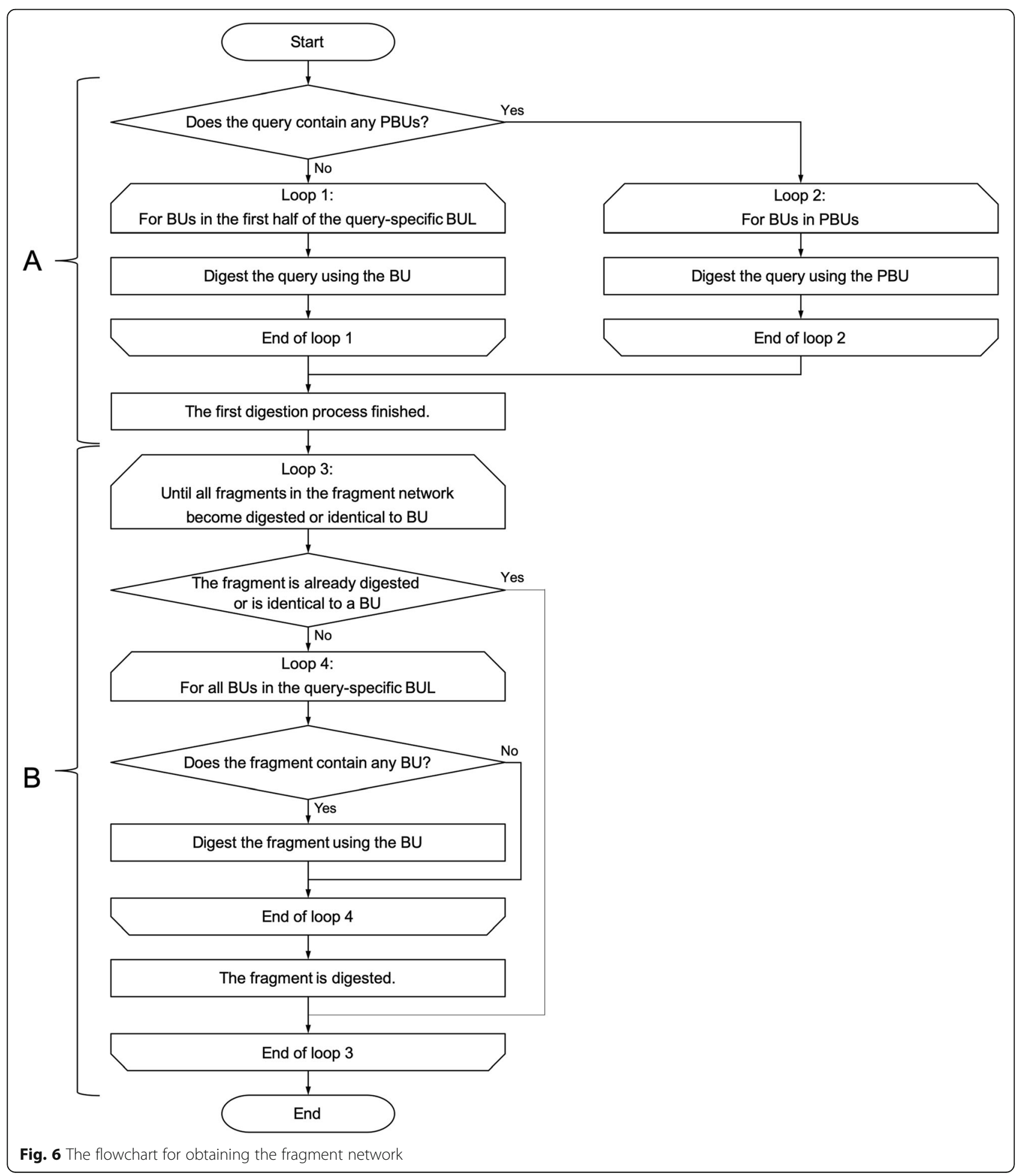


less than the sum of BBUs and DBUs because there were some common structures.

Some substructures, such as the glucose residue in glucosides, remain unchanged downstream in the biosynthetic pathway. To avoid an unnecessary increase in computational costs, we defined a set of PBUs that contained five monosaccharide residues, a shikimic acid moiety, and a betalamic acid moiety (Fig. 2).

\section{The metabolic disassembler workflow}

The workflow consists of five steps: (1) input the target molecule as a query, (2) generate a query-specific BUL, (3) generate the fragment network, (4) generate and sort the BU combination candidates, and (5) output the calculation result.

First, a user inputs the target molecule as a query. Acceptable file formats are Molfile, SMILES (Simplified

\section{A}

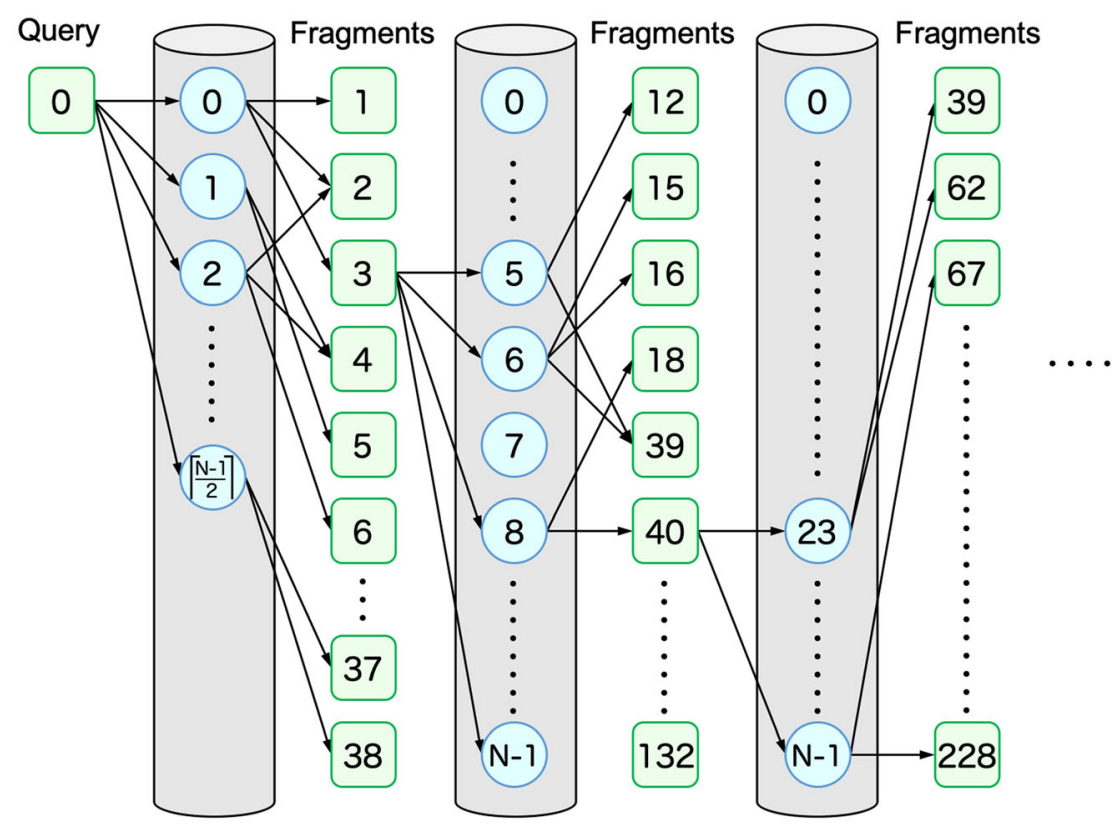

B

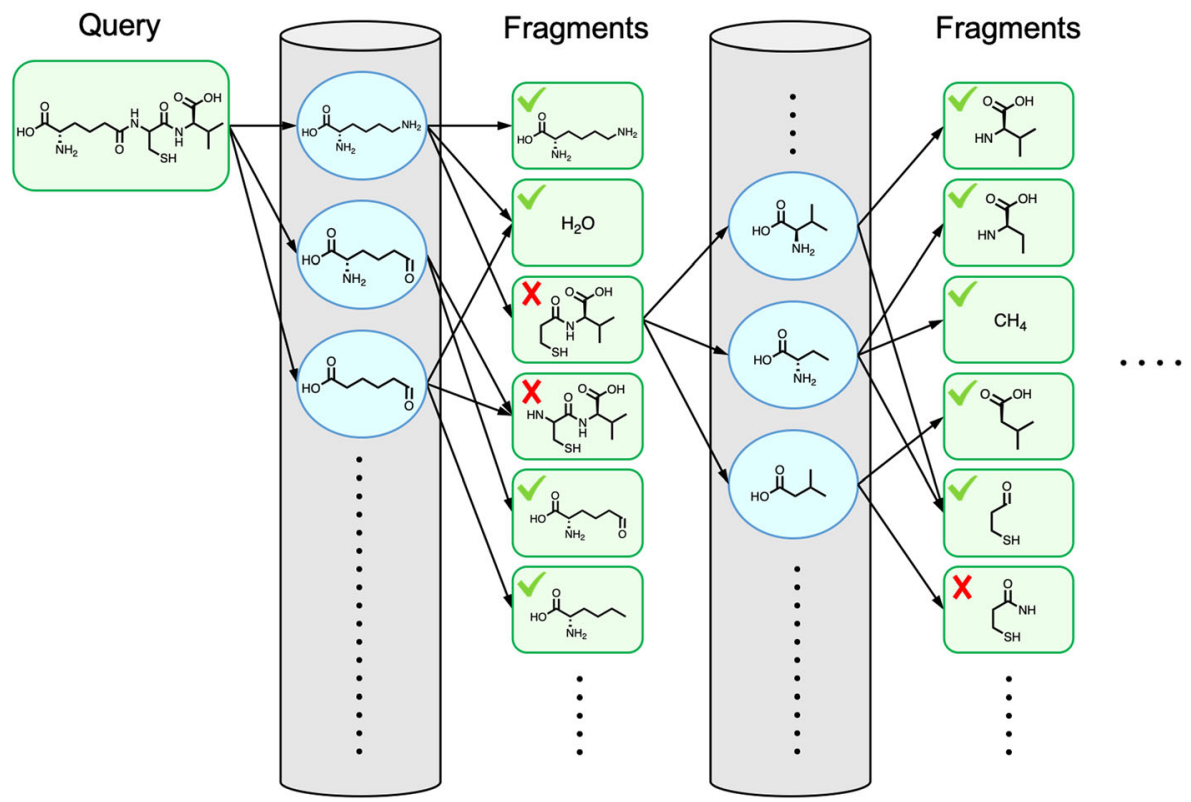

Fig. 7 Example fragment network. (Top) Schematic diagram where the same molecules or fragments are given the same IDs. (Bottom) The same diagram where the detailed chemical structures are described 
Molecular Input Line Entry System) [22], InChI (International Chemical Identifier) [23], and the KEGG compound identifier [13]. The input chemical structure is converted to the Mol object of RDKit [16] and is subsequently processed using NetworkX [18].

Second, each BU in the BUL is compared with the query structure. If the $\mathrm{BU}$ is part of the query structure, it is placed in the query-specific BUL. The aim of this process is to avoid unnecessary computation cost by removing BUs not included in the query molecule. The flowchart for generating a queryspecific BUL is shown in Fig. 3. We used the HasSubstructMatch method in the RDKit library with the following option:

rdkit.Chem.HasSubstructMatch((Mol)self, (Mol)query, useChirality $=$ True).
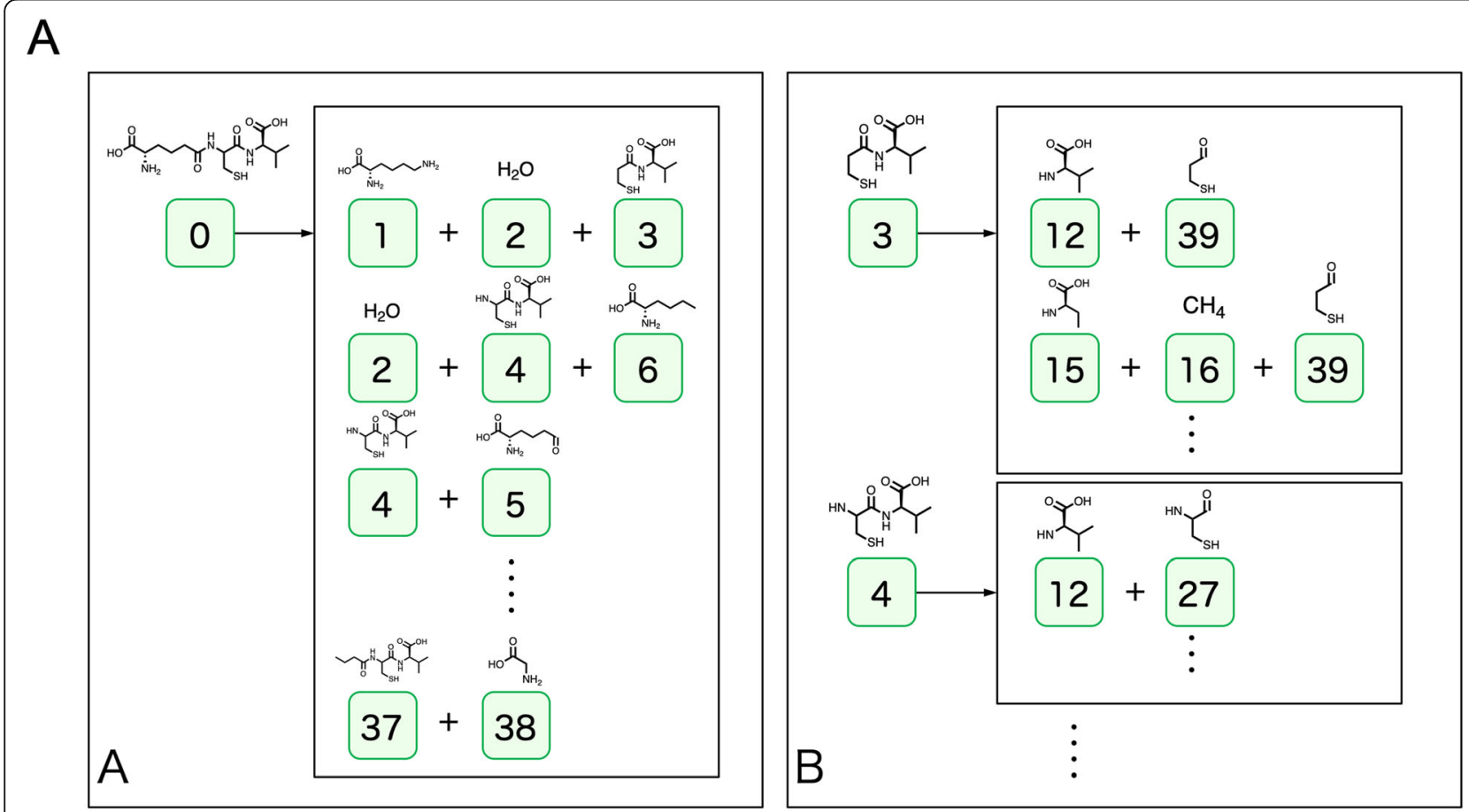

B

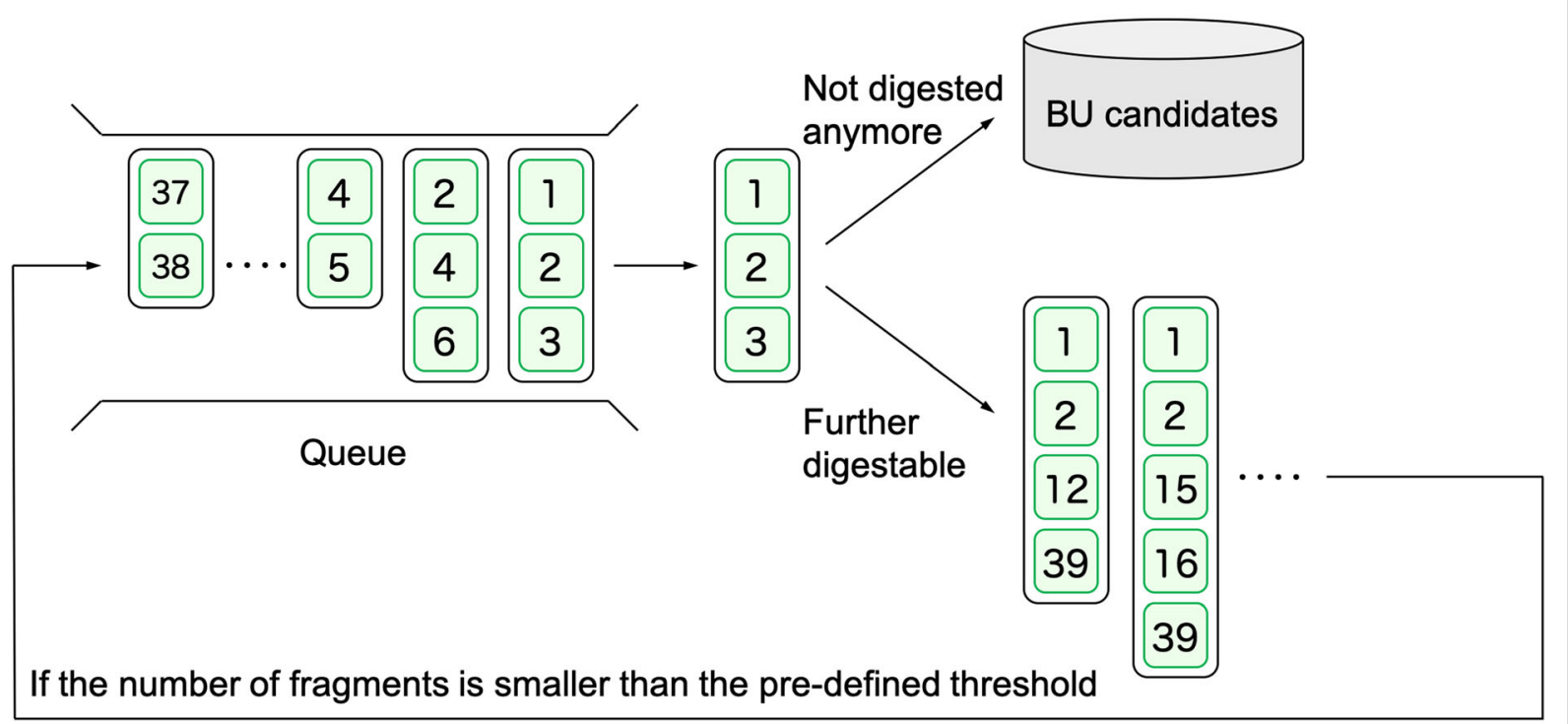

Fig. 8 Relationship of parent and child fragments and an example of the queue structure. (a) Example of the parent-child relationship from the fragment network. Panel A shows the initial fragmentation; panel B shows the further fragmentation. (b) Queue-based procedure to obtain BU candidates 
Table 1 Example of the sorted biosynthetic unit (BU) candidate list. The red dotted lines indicate the bond to be digested. NaN means there are no corresponding substructures or fragments. In this example, the top candidate is the best because it contains the largest fragment, and the number of the fragment is also the largest

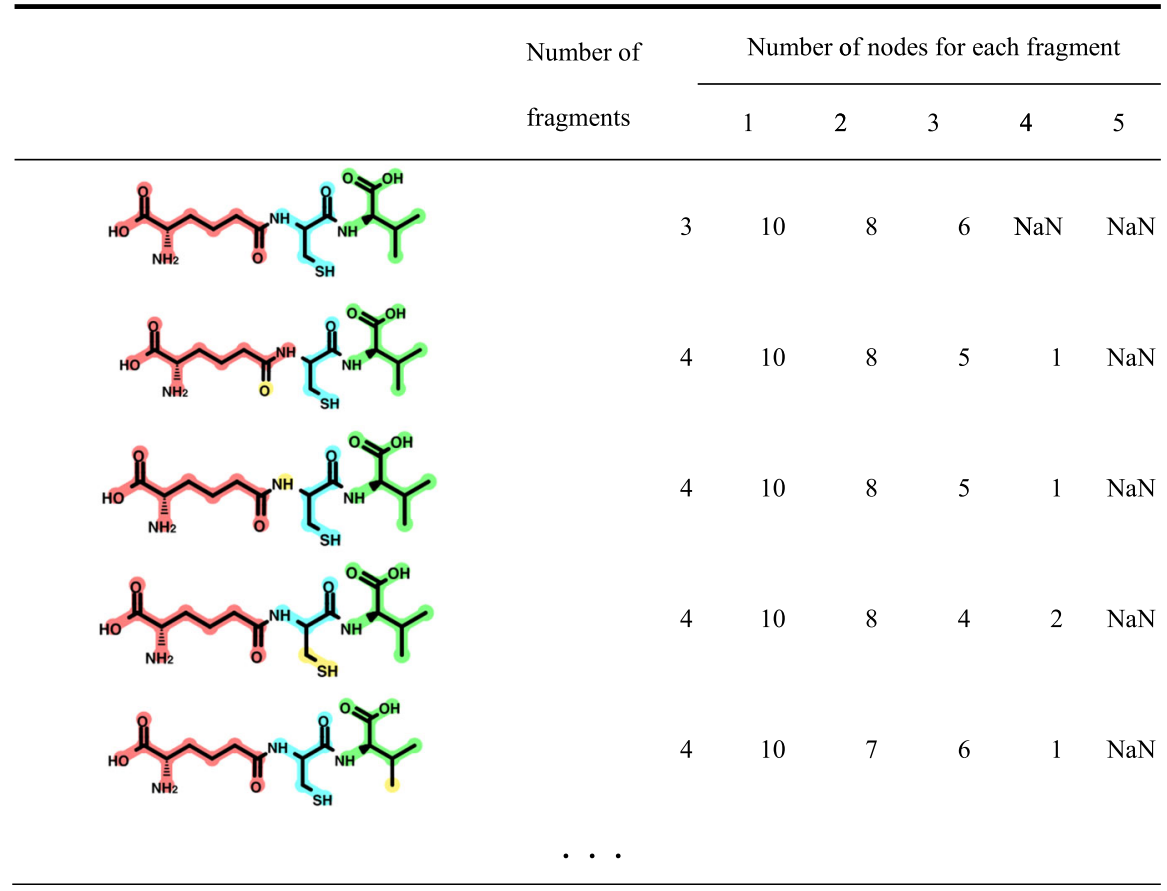

The red dotted lines indicate the bond to be digested. NaN means there are no corresponding substructures or fragments. In this example, the top candidate is the best because it contains the largest fragment, and the number of the fragment is also the largest

This method returns True if the query contains the given substructure, and returns False if not. Stereoisomers are distinguished, as shown in the example in Fig. 4a. Aromatic bonds are strictly distinguished from other conjugated double bonds, as shown in the example in Fig. 4b. This is an advantage in many cases; however, it is not useful in this study because aromatization and de-aromatization reactions are observed more frequently in biosynthetic reactions than in organic synthesis. To rescue such cases, we applied some empirical rules as follows. If the query molecule contains $\geq 17$ aromatic bonds, the Metabolic Disassembler uses the FindMCS method [16] and rescues the falsely judged substructures.

rdkit.Chem.rdFMCS.FindMCS $(\mathrm{mols}$, bondCompare = rdkit.Chem.rdFMCS.BondCompare.CompareAny).

The FindMCS method finds the maximum common substructure (MCS) in two or more molecules. Using the options as described above, bond orders such as aromatic and double bonds are ignored, thereby enabling an ambiguous MCS search. However, allowing too many ambiguous bonds produces an excessive number of candidate units. Therefore, the Metabolic Disassembler rescues a substructure only if the ratio of the number of ambiguous bonds to conjugated double bonds in the resulting MCS is $\leq 0.2$. After collecting the units for the query-specific BUL, the units are sorted in decreasing order of the number of the atoms they contain.

Third, the query molecule is divided repeatedly until every fragment matches a BU in the query-specific BUL, and a fragment network is generated to represent the relationship between the query molecule and the obtained fragments (Fig. 5). The fragment network is traversed to find the optimal combination of BUs. The MCS result is used to identify the bond that needs to be digested for fragmentation. The MCS for this process is:

rdkit.Chem.GetSubstructMatch((Mol)self, (Mol)query, useChirality $=$ True).

The flowchart used to obtain the fragment network has two stages (Fig. 6). In the first stage ("A" in Fig. 6), the first fragmentation is carried out by MCS against all the queryspecific BUs or PBUs to find the digested bond. The second stage ("B" in Fig. 6) consists of a nested loop, which repeats digestion until every fragment matches a BU. To reduce the number of candidate BU combinations, the maximum number of selected BUs is decided by eq. (1), where $\mathrm{N}$ is the total number of BUs in the query-specific BUL.

$$
\mathrm{n}=\lceil\mathrm{N} / 2\rceil(1) \text {. }
$$

An example of a fragment network produced by the above process is shown in Fig. 7. The fragment network 


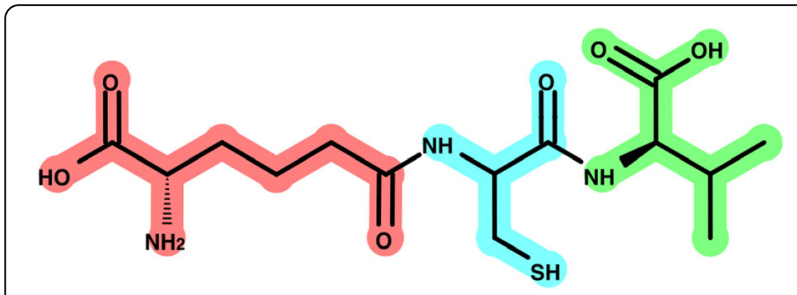

Fig. 9 Color-coded output representing the best fragmentation

represents the relationship between parent (i.e., before the split) and child (i.e., after the split) fragments. An example of this relationship is shown in Fig. 8a.

Fourth, using the parent-child relationship, a parent fragment can be replaced by child fragments, and this process can be iterated using the queue data structure (Fig. 8b). The fragment combination retrieved from the queue is checked to find whether it is identical to any one of the BUs in the query-specific BUL. If it is not, the replaced combination is again inserted into the queue structure as long as the number of fragments does not exceed the upper limit, which is determined in advance by the number of atoms included in the query molecule, as defined by eq. (2).

limit $\left._{\text {init }}=\Gamma \mathrm{N} / \mathrm{k}\right\rceil(2)$.

where, $\mathrm{k}=\{1$ if $N \leq 4 ; 3$ if $5 \leq N \leq 24 ; 4$ if $25 \leq N \leq 53$; 6 if $54 \leq \mathrm{N}\}$.

If every fragment is identical to one of the BUs in the query-specific BUL, the combination is added to the candidate list. The obtained candidate combinations are sorted in descending order of the size of the largest fragment as the first priority, and ascending order of the number of fragments as the second priority. An example of a sorted candidate list is shown in Table 1.

Finally, to easily understand the optimized BU combination, the Metabolic Disassembler outputs a colored image describing the fragmentation, as shown in the example in Fig. 9. Each of the split fragments is linked to the starting material.

\section{Comparison of the performance with the system that is not knowledge-based}

Since none of previous studies aimed to find starting materials of secondary metabolites directly, it is difficult to show the performance of our proposed workflow. Here, the performance of our proposed workflow, which is a knowledge-based system, was compared with that of a baseline approach using a genetic algorithm, which is not knowledge-based. We used the Python library named DEAP [24] (version 1.1.2) to implement the genetic algorithm. In the genetic algorithm, the disassembly of the query molecule into BUs was solved as an optimization problem of the digested bonds in the query molecule (Fig. 10), as explained below.

First, $\mathrm{N}$ individuals are generated randomly as the first generation, where an individual is represented as a binary ( 0 or 1$)$ vector that represents whether or not the corresponding bond should be digested. The fitness function decides whether an individual survives, and those that survive are subjected to any of three operations, namely the crossover of two individuals, mutation of an individual, or simply the copy of an individual. The generated $\mathrm{N}$ individuals are referred to as the next generation. This process is repeated until the predetermined ending condition is reached, and the individuals with highest fitness at the final generation are regarded as the best solution.

In this study, we set the population (the number of individuals in a generation) to 300 , the crossover probability to $80 \%$, and the mutation probability to $0.5 \%$. The fitness function was defined as the minimum similarity between the fragments and the BUs in the query-specific BUL. We used the Tanimoto coefficient of the Morgan fingerprint (radius 2, 1024 bit) [25] as the similarity measure.

\section{Results}

Performance evaluation

A summary of the ratio of correct disassembly for the respective pathway maps is shown in Table 2 . Note that

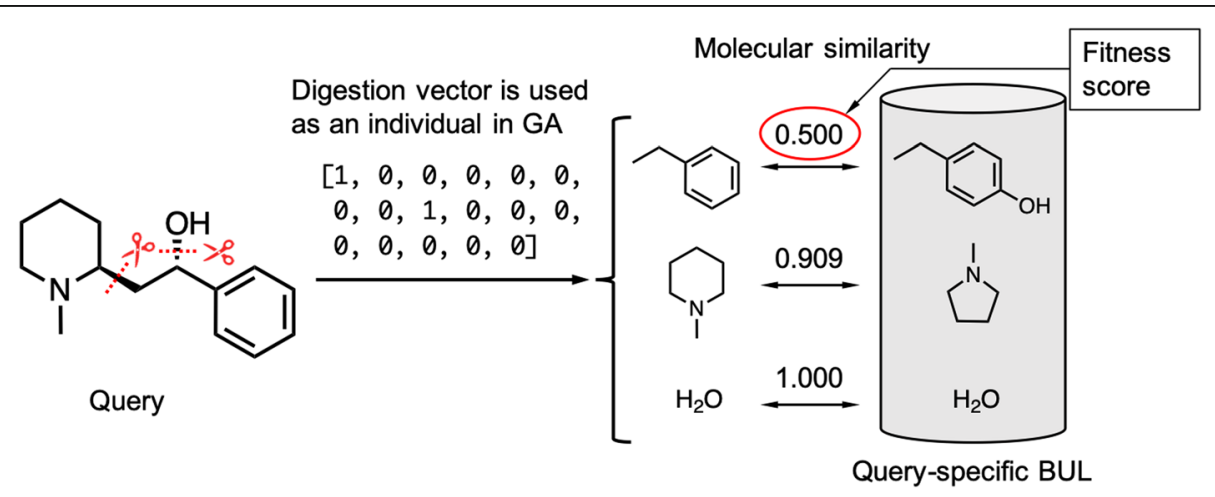

Fig. 10 Baseline workflow using the genetic algorithm. An individual represents the combination of digested bonds, and the fitness function was defined as the minimum similarity between the fragments and the BUs in the query-specific BUL 


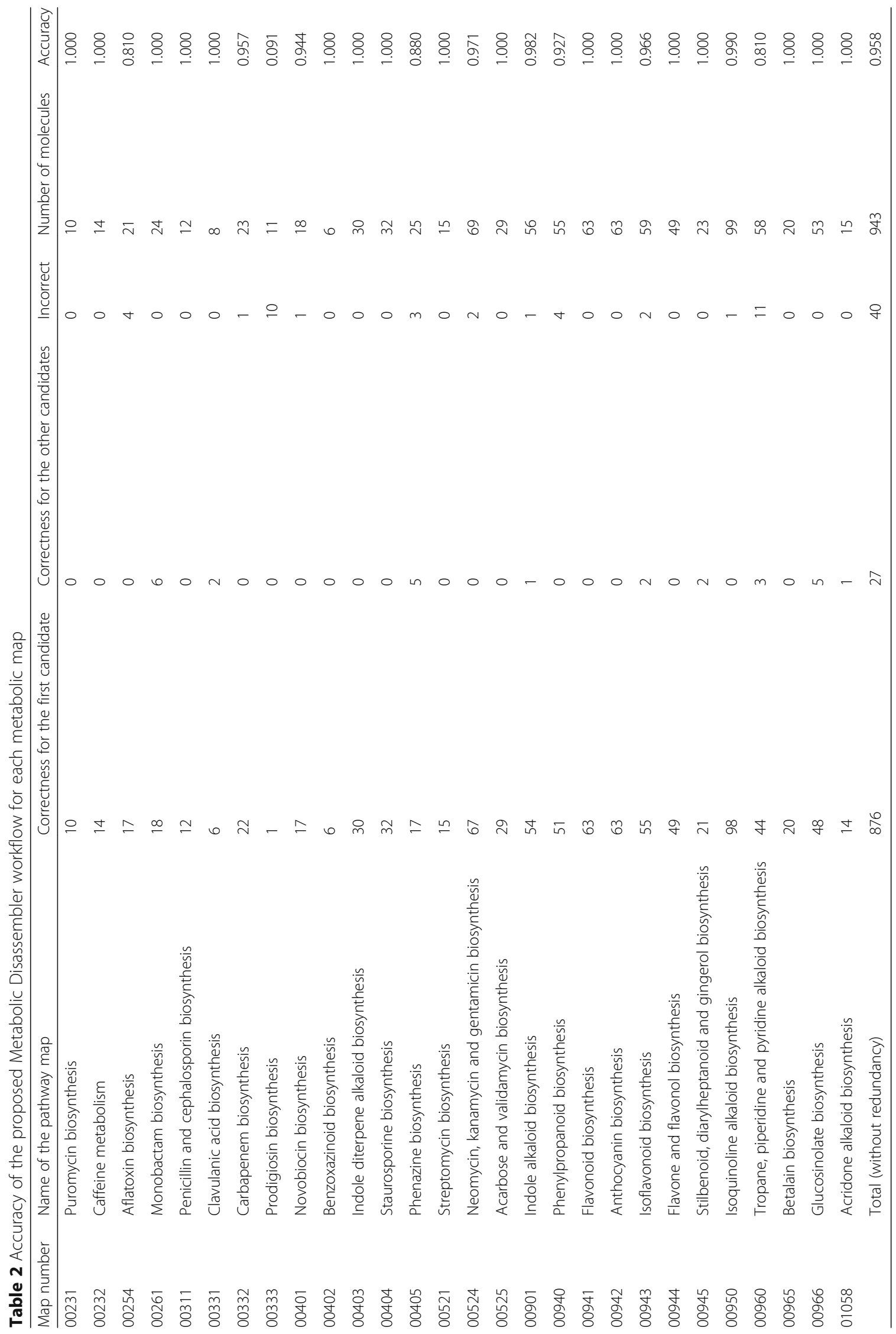


Table 3 Distribution of computational times for correct and incorrect disassembly

\begin{tabular}{llllll}
\hline & \multicolumn{5}{l}{ Runtime $(\mathrm{s})$} \\
\cline { 2 - 6 } & Average & Median & SD & Max & Min \\
\hline Correct answers $(n=903)$ & 27.5 & 2.0 & 121.0 & 2566.0 & 0.3 \\
Incorrect answers $(n=39)$ & 4.0 & 1.2 & 7.8 & 37.1 & 0.1 \\
All $(n=942)$ & 26.5 & 2.0 & 118.6 & 2566.0 & 0.1 \\
\hline
\end{tabular}

the Metabolic Disassembler inputs only the chemical structure of the query molecule and does not require any pathway information. We used the pathway maps only for the performance evaluation. We found that 903 of the 943 molecules (approximately 96\%) were disassembled correctly. Detailed results are presented in Additional file 1.

\section{Computational time}

We measured the computational time for the Metabolic Disassembler with the 943 molecules, executed in the computing environment described in Methods. We performed the calculation five times for each molecule and the obtained average values were recorded (Table 3). The plot of the computational time for each molecule is shown in Fig. 11. One of the molecules needed more than one day for the calculation, so it was excluded from the result. Therefore, the total number of molecules disassembled was 942. Among them, 855 molecules (approximately $91 \%$ ) were each disassembled within one minute, indicating the processing speed was sufficiently practical in the current implementation. Sixteen molecules took more than $5 \mathrm{~min}$ to disassemble (Table 4). One of the causes of the increased execution time came from the application of the FindMCS method in RDKit.
Comparison between the metabolic disassembler and the baseline workflow using a genetic algorithm

For the fair comparison to our proposed workflow, we measured the computational time of the baseline workflow and decided the number of generations to 200 , so that it takes approximately similar range of computational time. The average and maximum runtimes were 252 and $1512 \mathrm{~s}$, respectively, showing that the computational time was not different significantly from our proposed workflow. Also, in the evaluation process of the baseline workflow, we took top five most fitted individuals, and regarded as correct if one of them is correctly disassembled. Table 5 shows the comparison of our proposed workflow and the baseline workflow, and details of the numbers of correctly and incorrectly disassembled molecules using the baseline workflow are shown in Table 6. Among the molecules that were not correctly disassembled by the Metabolic Disassembler, five were correctly disassembled by the baseline workflow (Fig. 12). The baseline workflow does not depend on the distinction of aromatic bonds, which led to these successful cases. However, our proposed workflow generally performed better than the baseline workflow. The baseline workflow used the Tanimoto coefficient of Morgan fingerprint, which takes values from 0.0 (dissimilar) to 1.0 (similar). Importantly, 1.0 does not always mean identical. In addition, when the molecular similarity is low it is more difficult to interpret the obtained fragment. The proposed workflow performs exact matching, which provides easier interpretation.

\section{Discussion}

Our proposed workflow correctly disassembled 903 molecules, but incorrectly disassembled 40 molecules. The causes of the incorrect disassembly are given in Table 7.

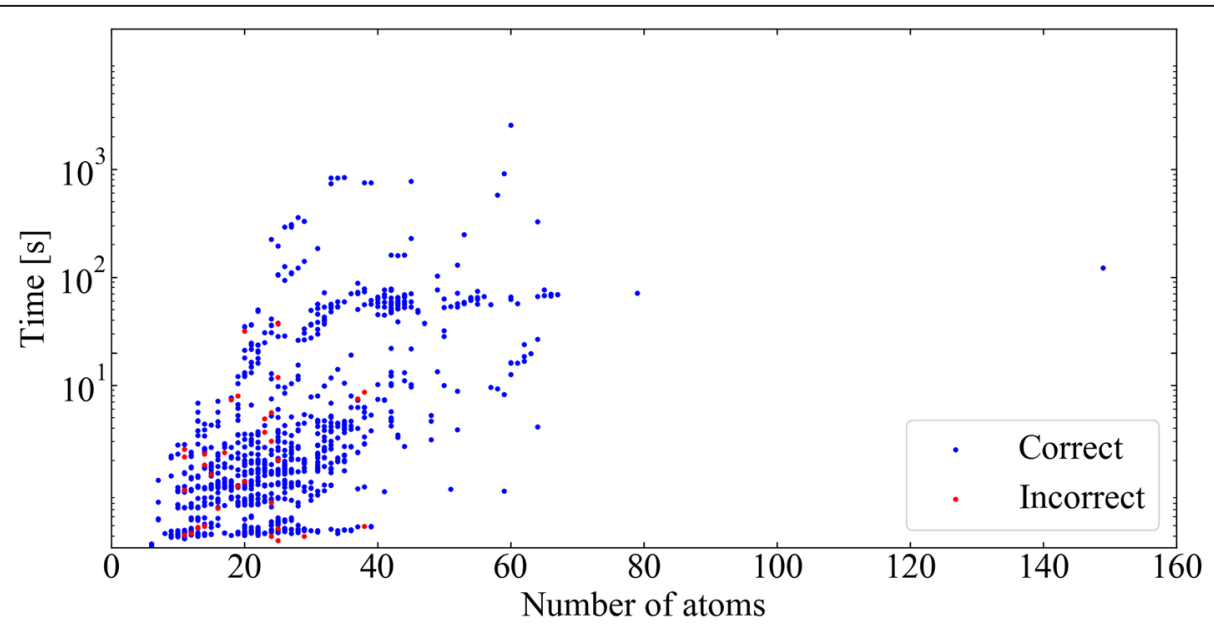

Fig. 11 Plot of computational time for each molecule. The computational time is given using a log scale 
Table 4 The molecules that needed more than five minutes of computational time for disassembly

Molecules

The substructure search methods caused most of the incorrect results. The HasSubstructMatch and GetSubstructMatch methods in RDKit were used for the substructure search, and these methods distinguish aromatic bond from conjugated double bonds. Our workflow allowed ambiguous bonds in limited circumstances, making it possible to cope with the metabolic reaction to form an aromatic ring by ring closure. However, three molecules, coumestrol (C10205), phenazine-1,6-dicarboxylic acid (C12119), and papaverine (C06533), were incorrectly disassembled. We decided not to apply this rule if the number of aromatic bonds in the query molecule was $\leq 16$ and because of this, six molecules, anhydroglycinol (C10200), 2-heptyl-4-quinolone (C20643), 2-heptyl-3-hydroxy-4-quinolone (C20643), coumarin (C05851), scopoletin (C01752), and scopolin (C01527), were incorrectly disassembled. Among the nine incorrectly disassembled molecules, coumarin, scopoletin, scopolin, coumestrol, and papaverine were correctly disassembled by the baseline approach, as explained above. However, the other four molecules were incorrectly disassembled by both workflows. We used the FindMCS method in RDKit to allow ambiguous matching, but this method easily leads to an increase in the calculation time and a decrease in interpretability. Additionally, the five molecules in map00960, slaframine (C06185), 13-(2-methylcrotonoy1)oxylupanine (C04170), 13-hydroxylupanine (C02621), pseudopelletierine (C10865), and calystegin A3 (C10850), were incorrectly disassembled despite of the presence of relevant BUs. This was caused by the GetSubstructMatch method in RDKit, which returns only the first found substructure even if theoretically there are more substructures, as shown in the example in Fig. 13. This problem could be solved by using GetSubstructMatches in RDKit, which returns all possible combinations, instead of GetSubstructMatch. However, this method easily causes an increase in the calculation time and, thus, it is not efficient enough to be applied in a simple way. Therefore, the future improvement of these problems would include the development of a more suitable method for the substructure search.

Some of the other unsuccessful disassembles were caused by rearrangements of the ring structures. For example, the structures of the fused rings in aflatoxin B1 (C06800), B2 (C16753), G1 (C16755), and G2 (C16754) were different from those of the respective precursors, which caused the incorrect disassembly. This problem could be remedied by adding more substructures to the BUL; however, this is not realistic.

We introduced PBUs with the aim of shortening the calculation time, especially for molecules that contain sugar residues. We also compared the algorithms that did and did not use PBUs, taking lampranthin II (C08552), which has a betalamic acid (C08538) moiety and saccharide residues, as an example (Fig. 14a). The 
Table 5 Comparison of the performance of the proposed Metabolic Disassembler workflow and the baseline workflows

\begin{tabular}{llll}
\hline & Correct & Incorrect & Accuracy \\
\hline Proposed workflow & 903 & 40 & 0.958 \\
$\begin{array}{l}\text { Baseline workflow using } \\
\text { the genetic algorithm }\end{array}$ & 490 & 453 & 0.520 \\
\hline
\end{tabular}

computational time was measured five times both before and after the introduction of the PBUs. The computational times before and after the introduction of the PBUs were $208.58 \pm 2.46 \mathrm{~s}$ and $4.38 \pm 0.11 \mathrm{~s}$, respectively; therefore, we concluded that introducing the PBUs significantly improved the computational speed. We found a case where introducing the PBUs led to an incorrect disassembly (Fig. 14b, c); however, this is the only incorrect case that we found, implying this was not a major problem.

In this study, we did not focus on terpenoids and polyketides, which were mainly in the metabolism map 1.9 class. The appropriate BUs of terpenoids and polyketides would be acetyl-CoA and so on, however, adding these BUs could not solve the problem. In this and other studies, manual curations are important for good performance. BBUs and DBUs were manually selected in order to appropriately disassemble molecules, and PBUs were selected in order to shorten the unnecessary computational time. Enhancement of the BUL could improve the coverage but may

Table 6 Accuracy of the baseline workflow for each metabolic map

\begin{tabular}{|c|c|c|c|c|c|}
\hline Map number & Name of the pathway map & Correct & Incorrect & Number of molecules & Accuracy \\
\hline 00231 & Puromycin biosynthesis & 4 & 6 & 10 & 0.400 \\
\hline 00232 & Caffeine metabolism & 11 & 3 & 14 & 0.786 \\
\hline 00254 & Aflatoxin biosynthesis & 13 & 8 & 21 & 0.619 \\
\hline 00261 & Monobactam biosynthesis & 9 & 15 & 24 & 0.375 \\
\hline 00311 & Penicillin and cephalosporin biosynthesis & 8 & 4 & 12 & 0.667 \\
\hline 00331 & Clavulanic acid biosynthesis & 8 & 0 & 8 & 1.000 \\
\hline 00332 & Carbapenem biosynthesis & 13 & 10 & 23 & 0.565 \\
\hline 00333 & Prodigiosin biosynthesis & 1 & 10 & 11 & 0.091 \\
\hline 00401 & Novobiocin biosynthesis & 13 & 5 & 18 & 0.722 \\
\hline 00402 & Benzoxazinoid biosynthesis & 6 & 0 & 6 & 1.000 \\
\hline 00403 & Indole diterpene alkaloid biosynthesis & 13 & 17 & 30 & 0.433 \\
\hline 00404 & Staurosporine biosynthesis & 11 & 21 & 32 & 0.344 \\
\hline 00405 & Phenazine biosynthesis & 4 & 21 & 25 & 0.160 \\
\hline 00521 & Streptomycin biosynthesis & 4 & 11 & 15 & 0.267 \\
\hline 00524 & $\begin{array}{l}\text { Neomycin, kanamycin and gentamicin } \\
\text { biosynthesis }\end{array}$ & 39 & 30 & 69 & 0.565 \\
\hline 00525 & Acarbose and validamycin biosynthesis & 20 & 9 & 29 & 0.690 \\
\hline 00901 & Indole alkaloid biosynthesis & 30 & 26 & 56 & 0.536 \\
\hline 00940 & Phenylpropanoid biosynthesis & 25 & 30 & 55 & 0.455 \\
\hline 00941 & Flavonoid biosynthesis & 42 & 21 & 63 & 0.667 \\
\hline 00942 & Anthocyanin biosynthesis & 32 & 31 & 63 & 0.508 \\
\hline 00943 & Isoflavonoid biosynthesis & 32 & 27 & 59 & 0.542 \\
\hline 00944 & Flavone and flavonol biosynthesis & 37 & 12 & 49 & 0.755 \\
\hline 00945 & $\begin{array}{l}\text { Stilbenoid, diarylheptanoid and gingerol } \\
\text { biosynthesis }\end{array}$ & 10 & 13 & 23 & 0.435 \\
\hline 00950 & Isoquinoline alkaloid biosynthesis & 31 & 68 & 99 & 0.313 \\
\hline 00960 & $\begin{array}{l}\text { Tropane, piperidine and pyridine alkaloid } \\
\text { biosynthesis }\end{array}$ & 16 & 42 & 58 & 0.276 \\
\hline 00965 & Betalain biosynthesis & 7 & 13 & 20 & 0.350 \\
\hline 00966 & Glucosinolate biosynthesis & 47 & 6 & 53 & 0.887 \\
\hline \multirow[t]{2}{*}{01058} & Acridone alkaloid biosynthesis & 12 & 3 & 15 & 0.800 \\
\hline & Total (without redundancy) & 490 & 453 & 943 & 0.520 \\
\hline
\end{tabular}




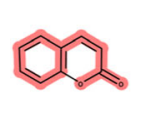

Coumarin

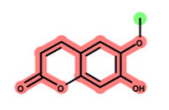

Scopoletin

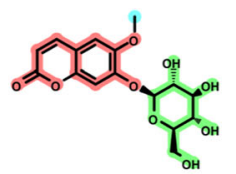

Scopolin

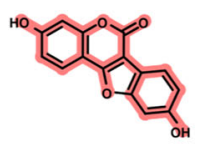

Coumestrol

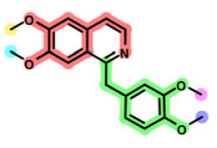

Papaverine

Fig. 12 The molecules that were correctly disassembled by the baseline workflow but not by the proposed workflow

increase the incorrect substructure matches. We already examined our manual curation, and the current BUs are the best at this moment. When it becomes apparent that some other secondary metabolites originate from other pathways, the enhancement of BUs may work well.

In fact, the 1.10 class also contains some terpenoids and polyketides and our proposed workflow produced incorrect disassembles for them. The four molecules in map000333, 2-methyl-3-n-amyl-dihydropyrrole (C21571), 2-methyl-3-namyl-pyrrole (C21572), 4-keto-2-undecylpyrroline (C21573), and 2-undecylpyrrole (C21574), are generated by amination and cyclization of fatty acids, but do not originate from Lproline as incorrectly indicated by our proposed workflow. Similarly, for the fatty acid dodecanoic acid (C02679) in map000333, the desired combination is the six $\mathrm{C}_{2}$ units that originate from malonyl-CoA (C00083). However, this molecule was excluded from the calculation because the number of fragments exceeded the upper limit.

The BUL could not support cases where the number of atoms decreased in the $\mathrm{BU}$ or when a double bond was generated in a BU by cyclization and subsequent oxidation. For example, to cope with the $\mathrm{C}_{7}$ units derived from octanoyl-CoA (C01944), it is necessary to prepare 19 BUs in which one of the six C$\mathrm{C}$ bonds becomes a double bond. There are 33 combinations for the $\mathrm{C}_{8}$ units. Therefore, preparing for other $C_{n}$ units produces an enormous number of BUs and slows the processing speed. In addition, if many straight-chain alkyl groups are registered in the BUL, molecules that did not originate from acetate-malonic acid pathway are adversely affected. Therefore, we concluded that the BUL and our proposed workflow should not be applied to the biosynthesis of terpenoids and polyketides.

Despite the efforts to enhance the BUL to cover a wide range of natural products, there were still nine molecules that were not covered. Apramycin (C01555) and oxyapramycin (C17997) in map00524 are synthesized via paromamine (C01743; Fig. 15a). These two molecules look like dimers but not exactly, and their biosynthetic pathways are not yet apparent. They contain substructures other than the paromamine (C01743) residue and their origins are unclear, and could not be dealt with by the proposed workflow. 3-(2-Carboxyethenyl)-cis,cis-muconate (C04366) in map00940 is synthetized by oxidative cleavage of the benzene ring of caffeic acid (C01197; Fig. 15b). This molecule can be dealt with by preparing a relevant $\mathrm{BU}$, but such cleavage was observed only in this molecule. Therefore, we decided we should not prepare such a BU considering its low general use. Senecionine (C06176) and senecionine $\mathrm{N}$-oxide (C15612) in map00960 are synthesized by condensation of retronecine (C06177) and two L-isoleucine (C00407; Fig. 15c). A carbon atom from each L-isoleucine unit is eliminated

Table 7 Causes of incorrect disassembly

\begin{tabular}{|c|c|c|c|c|c|c|c|}
\hline Map number & MCS search & Change in ring structures & Use of PBUs & Fatty acid and polyketide biosynthesis & Shortage of BUs & Others & Total \\
\hline 00254 & 0 & 4 & 0 & 0 & 0 & 0 & 4 \\
\hline 00332 & 0 & 0 & 0 & 0 & 0 & 1 & 1 \\
\hline 00333 & 5 & 0 & 0 & 5 & 0 & 0 & 10 \\
\hline 00401 & 0 & 0 & 0 & 0 & 0 & 1 & 1 \\
\hline 00405 & 3 & 0 & 0 & 0 & 0 & 0 & 3 \\
\hline 00524 & 0 & 0 & 0 & 0 & 2 & 0 & 2 \\
\hline 00901 & 0 & 0 & 1 & 0 & 0 & 0 & 1 \\
\hline 00940 & 3 & 0 & 0 & 0 & 1 & 0 & 4 \\
\hline 00943 & 2 & 0 & 0 & 0 & 0 & 0 & 2 \\
\hline 00950 & 1 & 0 & 0 & 0 & 0 & 0 & 1 \\
\hline 00960 & 5 & 0 & 0 & 0 & 6 & 0 & 11 \\
\hline Total & 19 & 4 & 1 & 5 & 9 & 2 & 40 \\
\hline
\end{tabular}




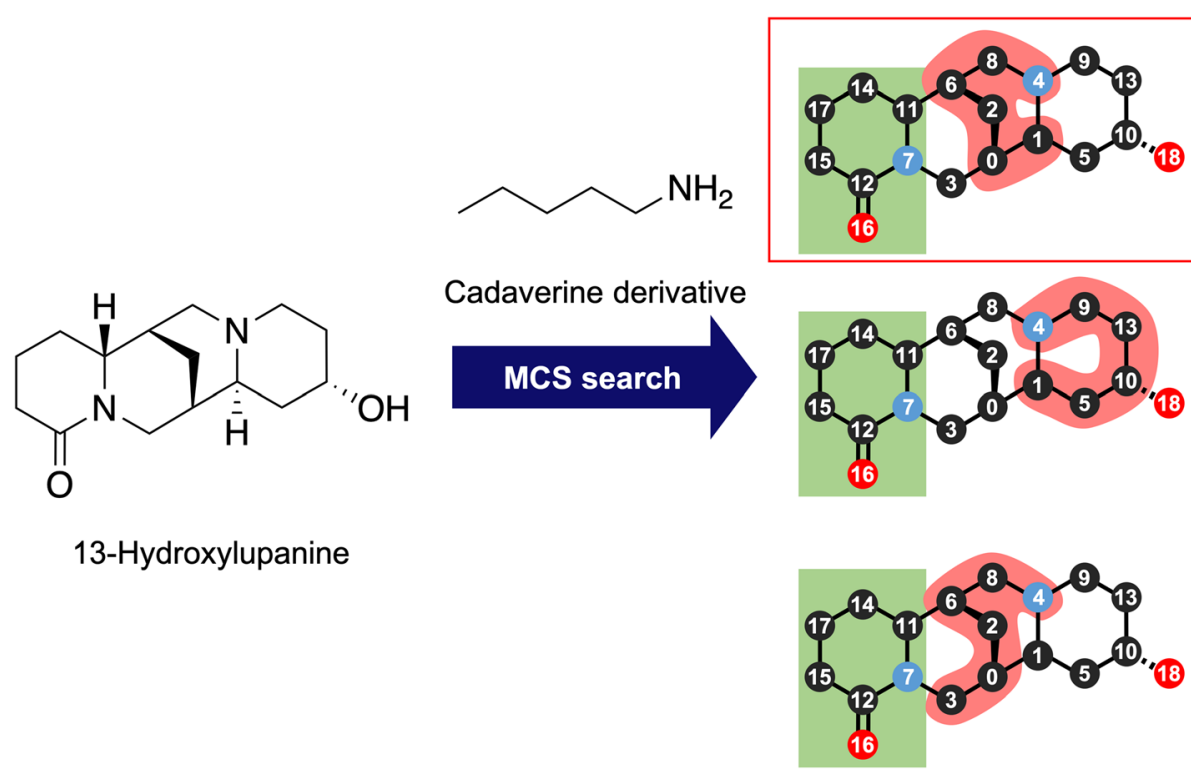

Fig. 13 Substructure search of cadaverine against 13-hydroxylupanine.The substructures highlighted in red correspond to the query cadaverine. The substructures in green rare those already matched to other substructures. The GetSubstructMatch method of RDKit returns only the first result found, even though theoretically there are more possible results

during the biosynthesis, but the reaction mechanism is not clear. Lobelanine (C10157), (-)-lobeline (C07475), and $(-)$-sedamine $(\mathrm{C} 10171)$ originate from piperideine (C06181), but their biosynthetic mechanisms are not clear (Fig. 15d). Cytisine (C10763) is biosynthesized by conjugating cadaverine (Fig. 15e); however, four carbon atoms are eliminated during the biosynthesis, but its complete biosynthetic mechanism has not been revealed. Therefore, appropriate BUs could not be prepared for these molecules.

Although enhancement of the BUL could be a solution, its expansion may increase the number of incorrect substructure matches as well as the calculation time. A sufficient number of BUs is necessary for successful disassembly, therefore, it is necessary to strengthen the BUL while considering its tradeoff.

\section{Conclusions}

The Metabolic Disassembler disassembles target chemical structures into relevant biosynthetic units that correspond to their starting materials, which is the first step in predicting the biosynthetic pathways of natural products. The users can use the Python program as well as the BUL so that the users do not have to reproduce this knowledge-based system. The Metabolic Disassembler will also help to identify the chemical bonds generated during the biosynthetic pathway, thereby providing valuable information for predicting the biosynthetic pathway of natural products.

\section{Supplementary information}

Supplementary information accompanies this paper at https://doi.org/10. 1186/s12859-019-3183-9.

Additional file 1. Summary of disassembly calculation for each map.

Detailed explanation of disassembly calculation for each pathway map.

\section{Abbreviations}

BBU: basic BU; BU: biosynthetic unit; BUL: biosynthetic unit library;

$\mathrm{DBU}$ : derivative $\mathrm{BU}$; $\mathrm{PBU}$ : preferential $\mathrm{BU}$

\section{Acknowledgments}

We thank Margaret Biswas, PhD, from Edanz Group (www.edanzediting.com/ac) for editing a draft of this manuscript.

\section{Authors' contributions}

KA conducted all calculations and most of the data curation, interpretation, and wrote the draft of manuscript. TM contributed to the data curation and interpretation. KT and KF gave helpful suggestions. MK designed the study, interpreted the results, and compiled the manuscript. All authors approved the final version of the manuscript.

\section{Funding}

This work was supported by the Japan Society for the Promotion of Science under the Grants-in-Aid for Scientific Research (KAKENHI) Program (grant number $17 \mathrm{~K} 07260)$.

\section{Availability of data and materials}

The Metabolic Disassembler is freely available at https://github.com/themetabolic-disassembler/metadisassembler.

Ethics approval and consent to participate Not applicable.

Consent for publication Not applicable. 
(a)

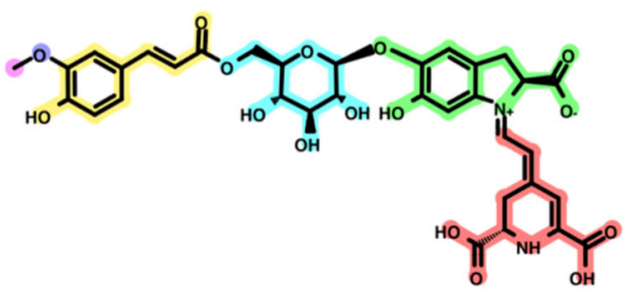

(b)<smiles>NCCc1c[nH]c2ccccc12</smiles>

Tryptamine

\section{Secologanin}

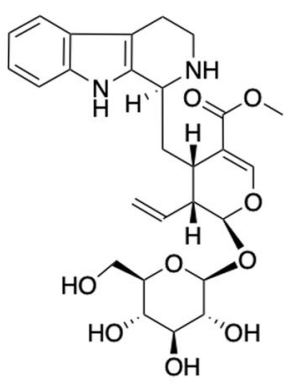

Strictosidine

(c)

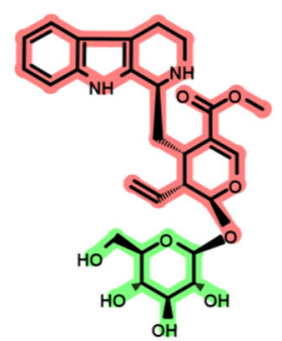

Fig. 14 Effects of preferential biosynthetic units on disassembly by the Metabolic Disassembler. (a) Disassembly of lampranthin II. (b) Biosynthesis of strictosidine. (c) Incorrect disassembly of strictosidine

\section{Competing interests}

None declared.

\section{Author details}

${ }^{1}$ School of Life Science and Technology, Tokyo Institute of Technology, 2-12-1 Ookayama, Meguro-ku, Tokyo 152-8550, Japan. ²Department of Chemical System Engineering, School of Engineering, The University of Tokyo, 7-3-1 Hongo, Bunkyo-ku, Tokyo 113-8656, Japan.

Received: 10 June 2019 Accepted: 30 October 2019

\section{1. - . - 0}

\section{References}

1. Fleming A. On the antibacterial action of cultures of a penicillium, with special reference to their use in the isolation of B. influenzae. Br J Exp Pathol. 1929;10(3):226-36.

2. Burg RW, Miller BM, Baker EE, Birnbaum J, Currie SA, Hartman R, Kong YL, Monaghan RL, Olson G, Putter I, Tunac JB, Wallick H, Stapley EO, Oiwa R, Omura S. Avermectins, new family of potent anthelmintic agents: producing organism and fermentation. Antimicrob Agents Chemother. 1979; 15(3):361-7.
3. Carbonell P, Parutto P, Herisson J, Pandit SB, Faulon JL. XTMS: pathway design in an eXTended metabolic space. Nucleic Acids Res. 2014;42(W1): W389-94.

4. Koch, M, Duigou, T, Carbonell, P, and Faulon, JL. Molecular structures enumeration and virtual screening in the chemical space with RetroPath2.0, Journal of cheminformatics. 2017;9(1):64.

5. Delépine B, Duigou T, Carbonell P, Faulon JL. RetroPath2.0: a retrosynthesis workflow for metabolic engineers. Metab Eng. 2018;45:158-70.

6. Sivakumar TV, Giri V, Park JH, Kim TY, Bhaduri A. ReactPRED: a tool to predict and analyze biochemical reactions. Bioinformatics. 2016;32(22):3522-4.

7. Kumar A, Wang L, Ng CY, Maranas CD. Pathway design using de novo steps through uncharted biochemical spaces. Nat Commun. 2018;9(1):184.

8. Yousofshahi M, Lee K, Hassoun S. Probabilistic pathway construction. Metab Eng. 2011;13(4):435-44.

9. Dewick PM. Medicinal natural products: a biosynthetic approach 3rd ed. Chichester, England: John Wiley \& Sons; 2009.

10. Yamanishi Y, Tabei Y, Kotera M. Metabolome-scale de novo pathway reconstruction using regioisomer-sensitive graph alignments. Bioinformatics. 2015;31(12):i161-70.

11. Yuan L, Tian Y, Ding S, Liu Y, Chen F, Zhang T, Tu W, Chen J, Hu QN. PrecursorFinder: a customized biosynthetic precursor explorer. Bioinformatics. 2019;35(9):1603-4. 
(a)

(b)
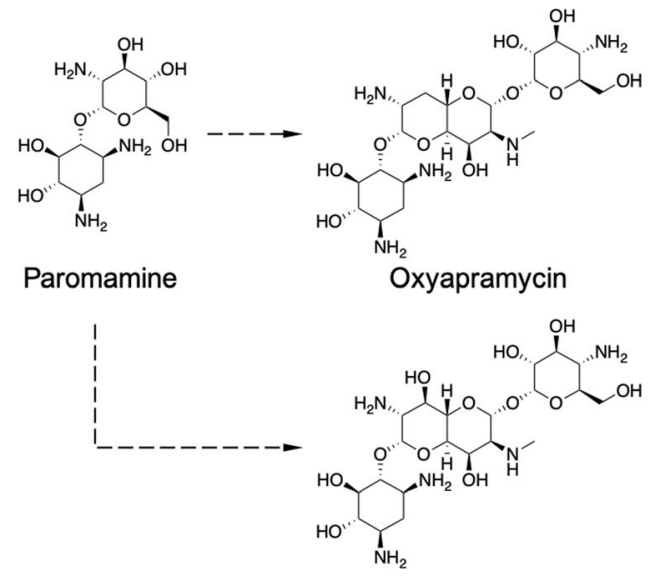

Apramycin

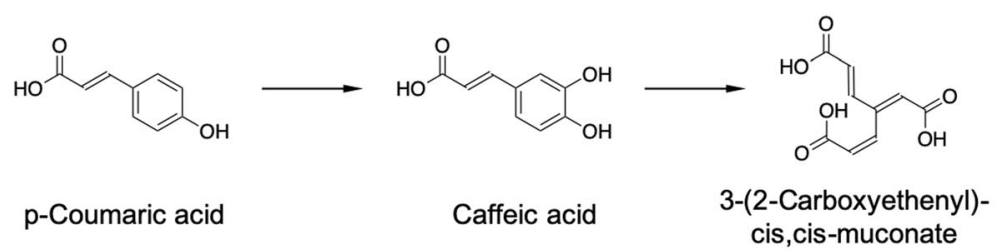

(c)

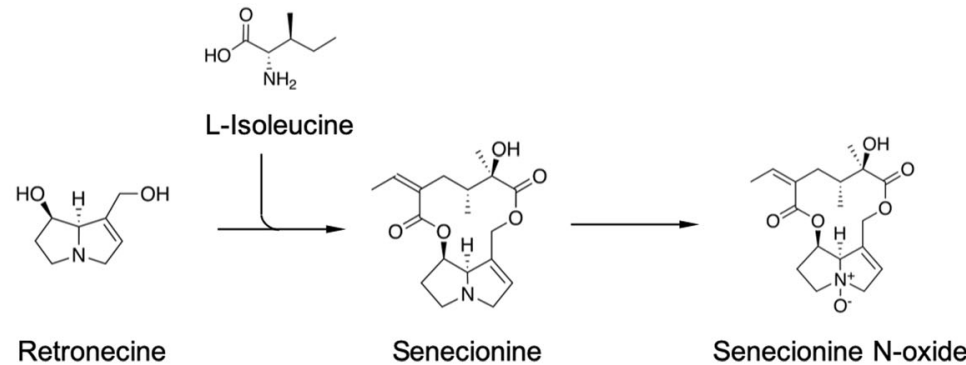

(d)
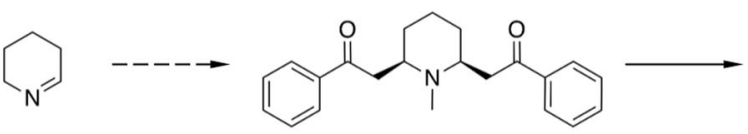

Piperideine

Lobelanine<smiles>CN1C(CC(=O)c2ccccc2)CCCC1C[C@H](O)c1ccccc1</smiles><smiles>[CH2-]C[IH]CI</smiles><smiles>CN1CCCCC1C[C@@H](O)c1ccccc1</smiles>

(-)-Sedamine

(e)

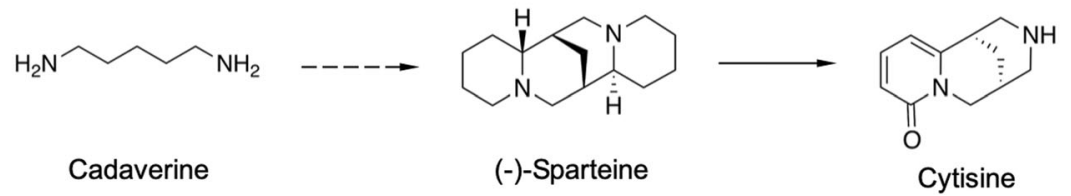

Fig. 15 Biosynthesis of the molecules that were not covered the BUL. (a) Apramycin and oxyapramycin. (b) 3-(2-carboxyethenyl)-cis,cis-muconate. (c) Senecionine. (d) Lobelanine and (-)-lobeline and (-)-sedamine. (e) Cytisine 
12. Moriya Y, Shigemizu D, Hattori M, Tokimatsu T, Kotera M, Goto S, Kanehisa M. PathPred: an enzyme-catalyzed metabolic pathway prediction server. Nucleic Acids Res. 2010;38:W138-43.

13. Kanehisa M, Goto S. KEGG: Kyoto encyclopedia of genes and genomes. Nucleic Acids Res. 2000;28(1):27-30.

14. Caspi R, Billington R, Fulcher CA, Keseler IM, Kothari A, Krummenacker M, Latendresse M, Midford PE, Ong WK, Paley S, Subhraveti P, Karp PD. BioCyc: a genomic and metabolic web portal with multiple omics analytical tools. The FASEB Journal. 2019;33(1_supplement):473-2.

15. Alcántara R, Axelsen KB, Morgat A, Belda E, Coudert E, Bridge A, Cao H, de Matos P, Ennis M, Turner S, Owen G, Bouqueleret L, Xenarios I, Steinbeck C. Rhea - a manually curated resource of biochemical reactions. Nucleic Acids Res. 2011;40(D1):D754-60.

16. RDKit: open-source cheminformatics, http://www.rdkit.org, Retrieved November 28, 2018

17. https://www.anaconda.com/

18. Hagberg A., Chult DS, and Swart P. Exploring network structure, dynamics, and function using NetworkX. Proc. 7th Python Sci. Conf. (SciPy 2008), Pasadena, California, United States. 2008;11-15.

19. https://cairographics.org/pycairo/

20. https://cairosvg.org

21. MDL Information Systems, CTFile formats, http://www.daylight.com/ meetings/mug05/Kappler/ctfile.pdf, 2003. Retrieved November 28, 2018

22. Weininger D. SMILES, a chemical language and information system. 1. Introduction to methodology and encoding rules. Journal of Chemical Information and Computer Sciences. 1988;28(1):31-6.

23. Heller S, McNaught A, Stein S, Tchekhovskoi D, Pletnev I. InChl - the worldwide chemical structure identifier standard. Journal of Cheminformatics. 2013;5(1):7.

24. Fortin FA, Rainville FMD, Gardner MA, Parizeau M, Gagné C. DEAP: evolutionary algorithms made easy. J Mach Learn Res. 2012;13:2171-5.

25. Rogers D, Hahn M. Extended-connectivity fingerprints. J Chem Inf Model. 2010:50(5):742-54.

\section{Publisher's Note}

Springer Nature remains neutral with regard to jurisdictional claims in published maps and institutional affiliations.

Ready to submit your research? Choose BMC and benefit from:

- fast, convenient online submission

- thorough peer review by experienced researchers in your field

- rapid publication on acceptance

- support for research data, including large and complex data types

- gold Open Access which fosters wider collaboration and increased citations

- maximum visibility for your research: over $100 \mathrm{M}$ website views per year

At $\mathrm{BMC}$, research is always in progress.

Learn more biomedcentral.com/submissions 